\title{
Tribute to a Distinguished Professor János Aczél at 85
}

Che Tat NG

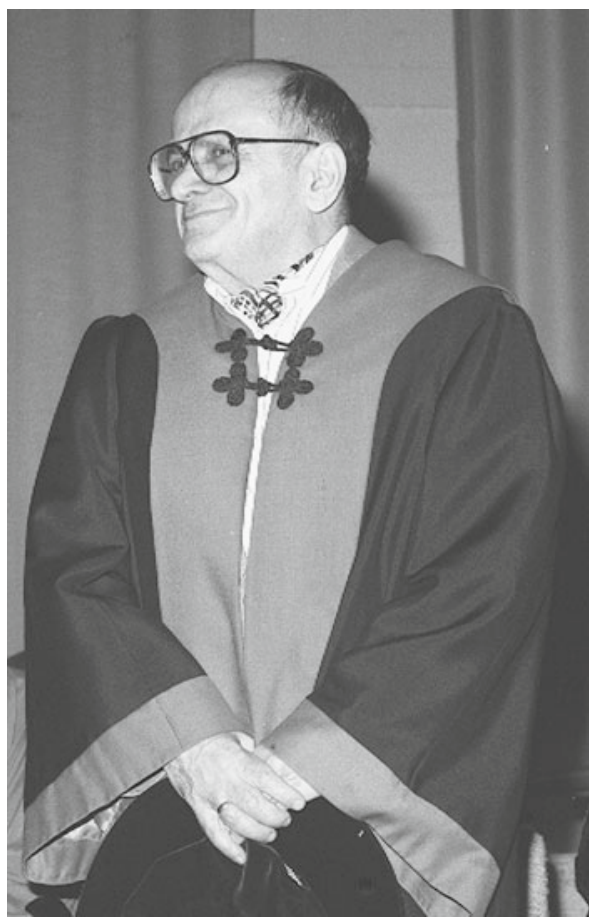

It is a pleasure for me to write this article devoted to János Aczél and his work. I thank the editors of Aequationes Mathematicae (AEM) for the invitation to do so on the occasion of his 85th birthday. Bruce Ebanks and I, on the occasion of his 80th birthday, wrote an article in AEM recounting some of our experiences with him when we were graduate students at Waterloo in the late sixties and early seventies. I shall attempt not to be overly repetitive.

I graduated from United College, the Chinese University of Hong Kong, in 1967, with a Bachelor's Degree in Mathematics. I stayed there as a 
Demonstrator in Mathematics for a year before coming to the University of Waterloo in 1968. Ling Fai Lam and I were the two Demonstrators in Mathematics. We came together to Waterloo for graduate studies. Ling Fai registered with the Department of Pure Mathematics and I was with the Department of Applied Analysis and Computer Science. It was Ling Fai who alerted me to the book [1] Lectures on Functional Equations and Their Applications, Academic Press, 1966 by János. Amazingly the book was available in the small library at United College. It is a most structured introduction to the subject.

János offered me a Research Assistantship. My duty was to help with the making of the bibliography index cards. I had the opportunity to see how he made a card for each published work in functional equations, labeled and tagged each according to its relevant classification index numbers, and wrote summary comments on it. I did some tagging, took the new cards to the data processing center for keypunching, and loaded the new data on reels of IBM tapes. The data could then be searched using the IBM 360 computer, or by hand using the paper cards and the colored tags. I learned how dedicated, organized and disciplined he was, and could associate that with the extensive bibliography found in the mentioned book which had an earlier German edition.

1968 was also the year AEM launched its first volume. A. M. Ostrowski was the Honorary Editor in Chief, János was the Editor in Chief and G. E. Cross was the Managing Editor. The Editorial Office was at Waterloo. The Faculty of Mathematics was very supportive in its launch. Undoubtedly, his books, the journal and the annual ISFE meetings he organized lay fertile ground for the subject to blossom.

In the early 1970s, Canada was in a deep and long recession. The mounting of bibliography data on tapes was relatively expensive. The computer tape search saw limited usage as many of us found papers we needed by just asking János and got instant reliable answers. The tape mounting came to an end. János and Susan continued with their building of the card system, and published the collected bibliography in AEM through a series of Works on Functional Equations starting with volume 1. Thanks to their initial efforts, a rich library of references was published sequentially in AEM and available to all researchers in the field [10].

His main interests are in functional equations in several variables. Some of his results are not about the solving of functional equations for their solutions per se, but about the hidden inter-connecting regularity properties of the functions satisfying some functional equations. For example he shows that if a real-valued $f$ satisfies the functional equation

$$
f(x+y)=F(x, f(y))
$$

for all real $x, y$ in some interval, then a continuous nonconstant $f$ must be strictly monotonic. It was reported in [5] and credit was given to [4]. Most of 
the time, he solved the underlying equations. In [6] he solved a conjecture of Srivastava and Srivastava regarding a characterization of the bivariate Poisson distributions by solving the equation

$$
G(p z+1-p, q w+1-q) G(p, q)=G(p z, q w)
$$

and obtaining the solution $G(z, w)=\exp \left[a_{1}(z-1)+a_{2}(w-1)\right]$ where $a_{1}, a_{2}$ are additive functions. He often strives to get the general solution without using regularity, and later offers some weak regularity properties which may restrict the solutions to the intended ones. For instance, continuity at some point is adequate to restrict the solution $G$ to those with linear $a_{1}$ and $a_{2}$.

The importance of the Cauchy type equations involving real variables rests with their frequent occurrences on the applied side. The build up of the theories around this class of equations is justifiably important. János has contributed much to enrich the field and to motivate many others to work in it. Thanks to the great collective effort put into it, equations which somehow make connection to the Cauchy type can be solved with relative ease and have broad applications.

In his work with Ostrowski [7] they solved the functional inequality

$$
\sum_{i=1}^{n} p_{k} f\left(q_{k}\right) \leq \sum_{i=1}^{n} p_{k} f\left(p_{k}\right)
$$

which holds for a fixed $n>2$ and for all $p_{k}>0, q_{k}>0$ with $\sum p_{k}=\sum q_{k}=1$. They showed that the solution is given by $f(p)=a \ln p+b$ for some constants $a \geq 0$ and $b$. The simple form of the solution could give the wrong impression that the result is easy to get by a connection to the Cauchy family. Only by reading the beautiful proof one begins to appreciate how much real analysis was used in the technical deductions.

If I remember correctly my first joint paper with János, written with Forte, was $[8]$.

Around the time Schweizer and Sklar published their book [11], motivated by problems in probabilistic metric space and in making group decisions, he joined forces with Alsina [3] and determined the class of quasi-arithmetic means $f$ satisfying the property $f\left(x_{1}^{p}, x_{2}^{p}, \ldots, x_{n}^{p}\right)=f\left(x_{1}, x_{2}, \ldots, x_{n}\right)^{p}$ for multiple values of $p$. They also characterized continuous t-norm $T$ satisfying $T\left(x^{p}, y^{p}\right)=$ $T(x, y)^{p}$ and illustrated how ideas used in the treatment of one topic could be shared by another.

As far back in time as 1955, he published a paper in Acta Math. Acad. Sci. Hung., volume 6 , on the translation equation $F(F(x, s), t)=F(x, s+t)$ along with the initial condition $F(x, 0)=x$. Assuming that $F$ is differentiable with respect to both variables $x$ and $t$, three differential equations arise together with differential initial conditions. Reich, [12], attributed the three equations to Jabotinsky. Our Austrian colleagues initiated much interest in their treatments. János and Gronau [2], for $x$ in a real or complex Banach space setting, 
and for $t$ in a real or complex neighbourhood of zero, examined each of the three Jabotinsky equations subject to various differential initial conditions.

One of his earliest works is on mean values [9]. His characterization of quasiarithmetic mean through bisymmetry is widely cited. In the book by Marley and Duncan Luce, Choice, Decision, and Measurement: Essays in Honor of R. Duncan Luce, Lawrence Erlbaum Associates, 1997, consistent aggregation and bisymmetry are featured.

With over 300 articles, 10 books and numerous honors, János remains as productive as ever and is a main source of inspiration.

On behalf of all the Editors of AEM, current and those who served in the past, I wish János a Happy Birthday!

\section{References}

[1] Aczél, J.: Lectures on Functional Equations and Their Applications. Academic Press, New York (1966)

[2] Aczél, J., Gronau, D.: Some differential equations related to iteration theory. Can. J. Math. XL, 695-717 (1988)

[3] Aczél, J., Alsina, C.: Characterizations of some classes of quasilinear functions with applications to triangular norms and to synthesizing judgements. Methods Oper. Res. 48, 3-22 (1984)

[4] Aczél, J., Kalmár, L., Mikusinski, J.: Sur l'équation de translation. Studia Math. 12, $112-116(1951)$

[5] Aczél, J.: On strict monotonicity of continuous solutions of certain types of functional equations. Can. Math. Bull. 9, 229-232 (1966)

[6] Aczél, J.: On a characterization of Poisson distributions. J. Appl. Probab. 9, 852-856 (1972)

[7] Aczél, J., Ostrowski, A.M.: On the characterization of Shannon's entropy by Shannon's inequality. J. Austral. Math. Soc. 16, 368-374 (1973)

[8] Aczél, J., Forte, B., Ng, C.T.: Why the Shannon and Hartley entropies are natural. Adv. Appl. Probab. 6, 131-146 (1974)

[9] Aczél, J.: On mean values. Bull. Am. Math. Soc. 54, 392-400 (1948)

[10] Forti, G.-L., Paganoni, L.: Works on functional equations and inequalities in several variables XXI. Aequationes Math. 74, 158-200 (2007)

[11] Schweizer, B., Sklar, A.: Probabilistic Metric Spaces. Elsevier/North Holland, New York (1982)

[12] Reich, L.: Holomorphe Lösungen der Differentialgleichung von E. Jabotinsky. Österr. Akad. Wiss. Math-Natur. Kl. Sitzungsber. II 195, 157-166 (1986)

Che Tat Ng

Department of Pure Mathematics

University of Waterloo

Waterloo, ON N2L 3G1, Canada

e-mail: ctng@math.uwaterloo.ca 